\title{
MICROMEGAS Signal: Numerical Simulation Based on Neon-Isobutane and Neon-DME
}

\author{
Hamid Mounir ${ }^{1}$, Seddik Bri² \\ ${ }^{1}$ Spectrometry Laboratory of Materials and Archaeomaterials (LASMAR), Faculty of Science, University \\ Moulay Ismail, Meknes, Morocco \\ ${ }^{2}$ Electrical Engineering Departments, High School of Technology, ESTM, University My Ismail, \\ Meknes, Morocco \\ Email: hamidmonir@gmail.com
}

Received 5 January 2015; accepted 23 January 2015; published 26 January 2015

Copyright (C) 2015 by authors and Scientific Research Publishing Inc.

This work is licensed under the Creative Commons Attribution International License (CC BY). http://creativecommons.org/licenses/by/4.0/

\section{(c) (i) Open Access}

\section{Abstract}

Recent years, we have seen the development of many fields of gas detectors. The MICROMEGAS (Micro-Mesh Gas Structure) appeared as the very promising detector. It is a major family of position detectors in High Energy Physics. This work is done in normal (NTP) based gas mixtures: neon are noble gas and isobutane and DME (dimethyl-ether) as moderators gas (quenchers), using ${ }^{55} \mathrm{Fe}$ as a radiation source (X-ray $5.9 \mathrm{keV}$ ). To address the modeling of MICROMEGAS detector, a descriptive model of different physical and geometrical phenomena MICROMEGAS was established by developing a simulation program to spreading the detector response. After, an analytical calculation of the potential and the electric field distributions has been presented briefly, to better estimate electrical and geometric configuration. Finally, simulation results of electrical signals based on gas mixtures (Neon-isobutane, Neon-DME) produced by MICROMEGAS were presented and analyzed in order to improve the MICROMEGAS performance (spatial $(12 \mu \mathrm{m})$ and temporal ( $0.7 \mathrm{~ns}$ ) resolutions).

\section{Keywords}

MICROMAEGAS, Gas Mixture, X-Ray Source, Avalanche Phenomenon, MATLAB Programing, Signal

\section{Introduction}

MICROMEGAS [1] is a high gain gaseous detector, which can stand up alone without a need of an additional pre-amplification. It is a new gaseous detector initially developed for track-in high-rate, high-energy, physics experiments since 1990. It shows higher counting rate capacity up to $10^{8} \mathrm{~mm}^{2} \cdot \mathrm{s}^{-1}$, position-sensitive with spatial resolution better than 100 microns and good performance of radiation hardness [2] [3], which has been developed since 1996 at SACLAY, France [4]. MICROMEGAS is a Parallel Plate Detector (PPD) with three elec- 
trodes, cathode, micromesh and anode and a narrow amplification space, typically 50 - 100 microns, between the micromesh and the anode.

The detector gain depends directly on the distance between the micromesh and anode; therefore, controlling the geometry of the amplification region is crucial in order to achieve good energy resolution and excellent timing properties [5]. These results were confirmed by a similar structure having wider amplification gap and thicker metallic grid [6].

\section{MICROMEGAS Description and Operating}

Detailed descriptions of MICROMEGAS are given in [1] [3] and [7]. A two-stage parallel plate avalanche chamber has a narrow amplification gap defined by the anode plane and a cathode plane made by Ni electroformed micromesh. Several 500 LPI, $5 \times 5 \mathrm{~cm}^{2}$ and of micro-mesh pitch $\left(p_{g}\right)$ with a conversion gap of $3 \mathrm{~mm}$, an amplification gap of $100 \mathrm{~mm}$ with a strip pitch of $317 \mathrm{~mm}$, have been designed and fabricated. The parallelism between the micromesh grid and the anode is maintaining by spacers of $150 \mathrm{~mm}$ in diameter and placing every 2 $\mathrm{mm}$. They are printedon a thin epoxy substrate by conventional lithography of a photo-resistive polyamide film. The thickness of the film defines the amplification gap. This cheap and simple process allows the construction of large detectors with excellent uniformity and energy resolution over the whole surface. Figure 1 shows geometric and physic descriptions of $\mu$-MEGAS detector.

\subsection{MCROMEGAS: Concept and Configuration}

The geometry of MICROMEGAS detector is then, reproduced by form of cuboids Figure 2(b). The coordinate system used is showing in Figure 2(a), where:

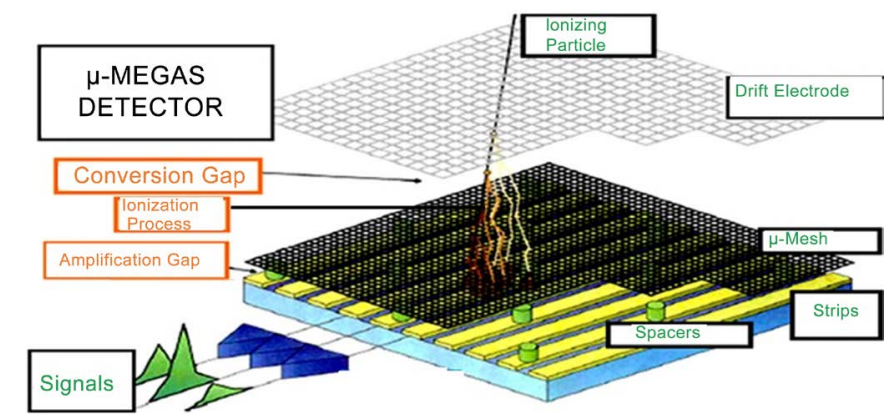

Figure 1. MICROMEGAS description: physical \& geometric processes.

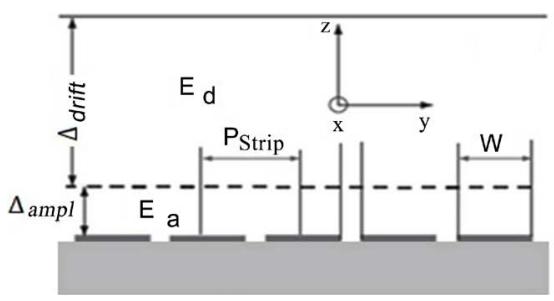

(a)

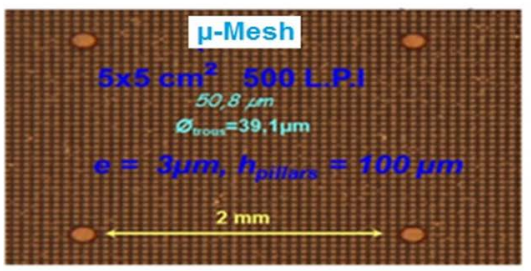

(c)

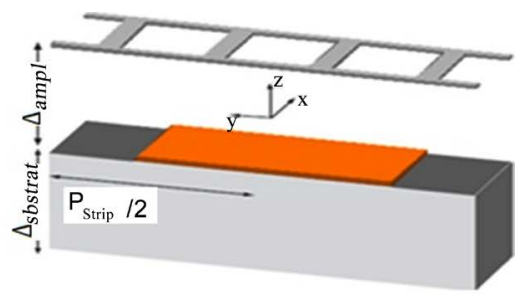

(b)

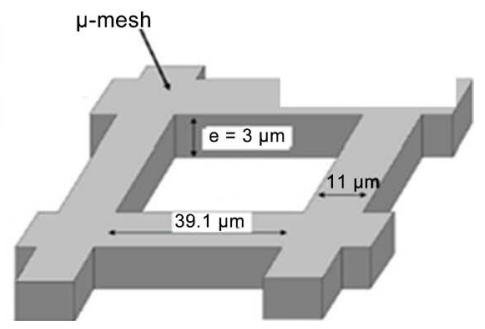

(d)

Figure 2. Coordinate system and geometric parameters (a) (b) and $\mu$-mesh prototype (c) (d) used in MICROMEGAS detector. 


$$
\left\{\begin{array}{l}
-\frac{p_{g}}{2} \leq x \leq \frac{p_{g}}{2} \\
-\frac{p_{\text {Strip }}}{2} \leq y \leq \frac{p_{\text {Strip }}}{2} \\
-\Delta_{\text {Substrat }} \leq z \leq \Delta_{\text {drift }}
\end{array} .\right.
$$

where $p_{g}$ is the Micro-Mesh pitch $p_{\text {strip }}$ is the strip pitch.

\subsection{Electric Field Configuration}

The knowledge of the shape of the electric field lines close to the micromesh is a key issue for an optimal operation of the detector and especially for an efficient transfer of electrons to the amplification gap. The electric field is homogeneous in both the conversion and the amplification gap. It exhibits a funnel like shape around the openings of the micro-grid: field lines are highly compressed towards the middle of the openings, into a small pathway equal to a few microns in diameter. The compression factor is directly proportional to the ratio of the electric fields between the two gaps. Figure 3 displays details of the field lines near the grid used in the present test.

The electrons liberated in the conversion gap by the ionizing radiation follow these lines and are focused into the multiplication gap where amplification process takes place. The ratio between the Electric Field in the amplification gap and that in the conversion gap must be set at large values $(>5)$ to permit a full electron transmission, and to reduce a part of ion cloud, produced in the avalanche, to escape into the conversion gap.

\subsection{Electric Field and Potential Distributions}

The mathematic term of potential is often calculated analytically using conformal transformations or SchwartzChritoffel transformation [7]. Our work is based on the use of Green functions [8]. The configuration that we want to study is presented in Figure 4.

After all calculus, considering all processes, we established the following form of Potential:

$$
\left\{\begin{array}{l}
V(y, z)=\int \rho(y, z) G_{D}\left(y, y^{\prime}, z, z^{\prime}\right) d y^{\prime} d z^{\prime}-\oint V\left(y^{\prime}\right) \frac{\partial G_{D}}{\partial z^{\prime}} d y^{\prime} \\
\phi(y, z)=\frac{V}{\pi}\left[\arctan \left(\cot \left(\frac{z \pi}{2 D}\right) \tanh \left(\pi \frac{y+w / 2}{2 D}\right)\right)-\arctan \left(\cot \left(\frac{z \pi}{2 D}\right) \tanh \left(\pi \frac{y-w / 2}{2 D}\right)\right)\right]
\end{array}\right.
$$

Figure 3. Profile of the electric field lines close to the $\mu$-Mesh in MICROMEGAS detector.

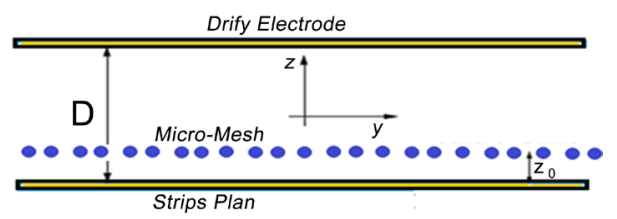

Figure 4. Configuring analytical calculation of the potential. 
where $\rho(y, z)$ is the charge density in $(y, z)$, and $G_{D}$ is the Dirichlet Function (Green Function) the weighting potential is presented in Figure 5.

The weighing field is deduced from Equation (2)

$$
\left\{\begin{array}{l}
E_{y}=V \frac{1}{2 D}\left[\frac{\sin \left(\frac{z \pi}{D}\right)}{\cosh \left(\pi \frac{y-w / 2}{D}\right)-\cos \left(\frac{z \pi}{D}\right)}-\frac{\sin \left(\frac{z \pi}{D}\right)}{\cosh \left(\pi \frac{y+w / 2}{D}\right)-\cos \left(\frac{z \pi}{D}\right)}\right] \\
E_{z}=V \frac{1}{2 D}\left[\frac{\sinh \left(\pi \frac{y-w / 2}{D}\right)}{\cosh \left(\pi \frac{y-w / 2}{D}\right)-\cos \left(\frac{z \pi}{D}\right)}-\frac{\sinh \left(\pi \frac{y+w / 2}{D}\right)}{\cosh \left(\pi \frac{y+w / 2}{D}\right)-\cos \left(\frac{z \pi}{D}\right)}\right] \\
E_{Y Z}=\sqrt{\left(E_{Y}^{2}+E_{Z}^{2}\right)}
\end{array}\right.
$$

Equations (2) and (3) have been implemented in a MATLAB program; we treat different cases related to the geometric shapes of a track to determine the optimal width and pitch of the tracks. The results of the $3 D$ potential and field distributions are shown in Figure 6 and Figure 7, respectively.

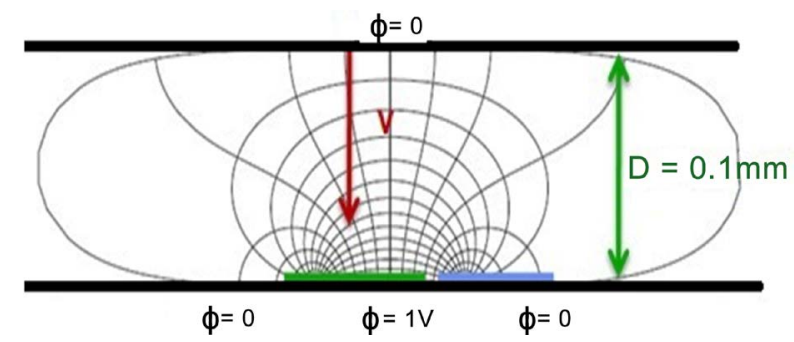

Figure 5. Weighting potential by applying 1 volt on a track $(\mathrm{V}=1 \mathrm{~V})$ and 0 volt on other.
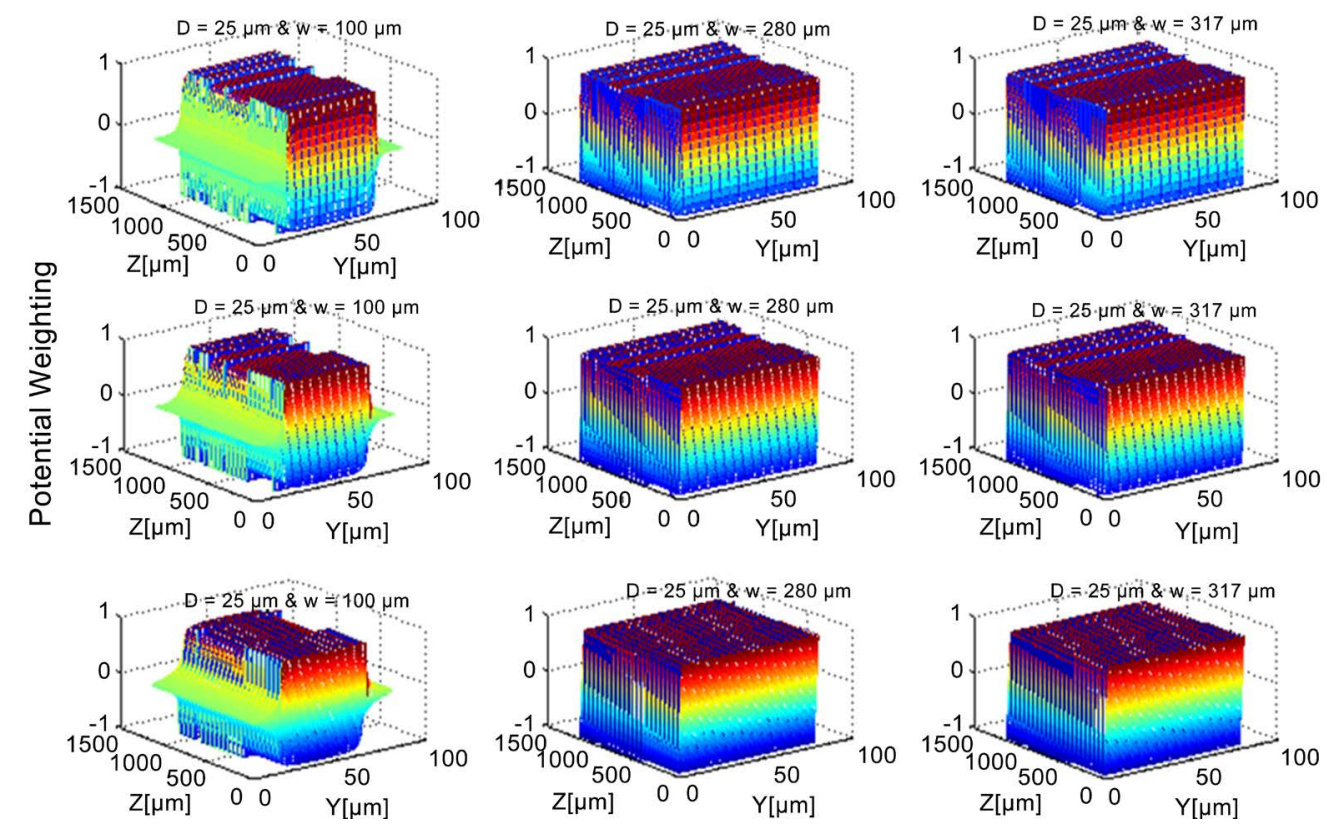

Figure 6. $3 D$ weighting potential distribution. 

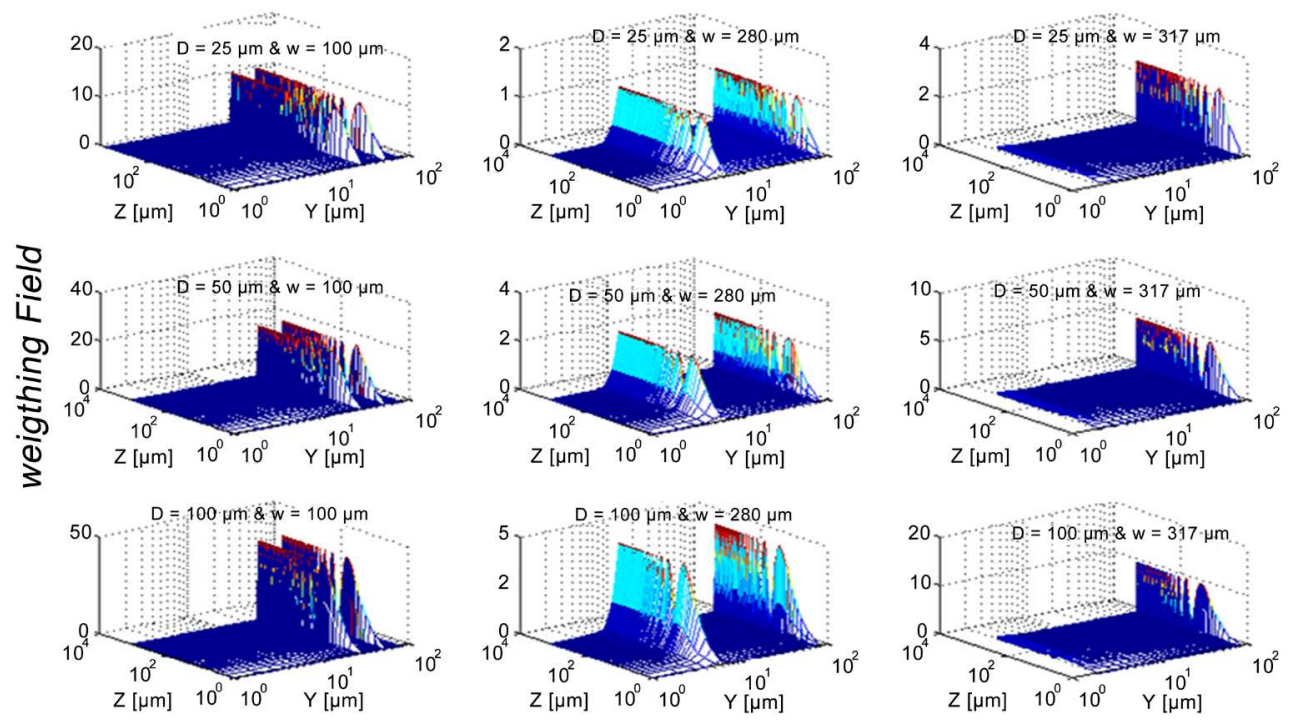

Figure 7. $3 D$ weighting field distribution.

Note that the estimate of the potential distribution is insufficient to determine the optimal width of the tracks to get a good configuration MICROMEGAS detector. But it seems that the configurations ( $w 1=280$ microns, $w 2=317$ microns and $D$ ) are satisfactory because the distributions are well presented. By against the corresponding distributions of the widths $w=100 \mu \mathrm{m}$ tracks, have signal distortion (deformation) which causes instabilities and system disturbances.

The distributions of different configurations of weighting field are shown in the figure above. Note that the distributions of peaks increase with the growth of $D$. Moreover, the peaks of the first column of the field distribution ( $w=100$ microns) are very close, which produces overlaps (spark between the wire of the tracks). The remaining columns have peaks with a larger spacing. It is concluded that sufficient choice of track width minimizes system instability, as an example of the third column ( $w=317$ microns), which has only one peak band which further improves the stability; have good performance (better resolution) to MICROMEGAS.

\section{Simulation Model}

The analytical study of our system leads us to extract some equations that lead us to model our detector described above. The simulation model of the physical processes occurring within MICROMEGAS is based on the MATLAB Program that is the tool of simulation, and more effective for our work. In the general case, there are three types of parameters affecting the detector: the parameters related to the chamber (geometry), the gas parameters (diffusion, gain, mixing coefficients), the parameters of the trace (angle, energy, signal, type of particles). Figure 8 shows the principle operating and the simulator used for MICROMEGAS detector modeling.

\subsection{Input Output Parameters Configuration and Characterization of MICROMEGAS}

Table 1 shows some input output parameters used in the MICROMEGAS detector modeling.

\subsection{Simulation of Charges Collected in MICROMEGAS}

From Equation (4) [9], [10] and Table 2 shown below [11], we can estimate the charge collected $Q_{i}(t)(I=1$ to 5$)$ in MICROMEGAS for different proportions of each gas mixture; using $\mathrm{X}$-rays $(5.9 \mathrm{keV})$ as a radiation source, the same program remains what we need to change the values of the coefficients $A_{i}$ and $B_{i}$ [11]-[13]. These parameters are adjusted by experience [14].

$$
Q(t)= \begin{cases}\frac{n_{0} q}{T_{w} d}\left(\exp \left(T_{w} \frac{d}{t_{e}} t\right)-1\right), & 0 \leq t \leq t_{e} \\ \frac{n_{0} q}{t_{\text {ion }}} \exp \left(T_{w} d\right) t, & t_{e} \leq t \leq t_{\text {ion }}\end{cases}
$$




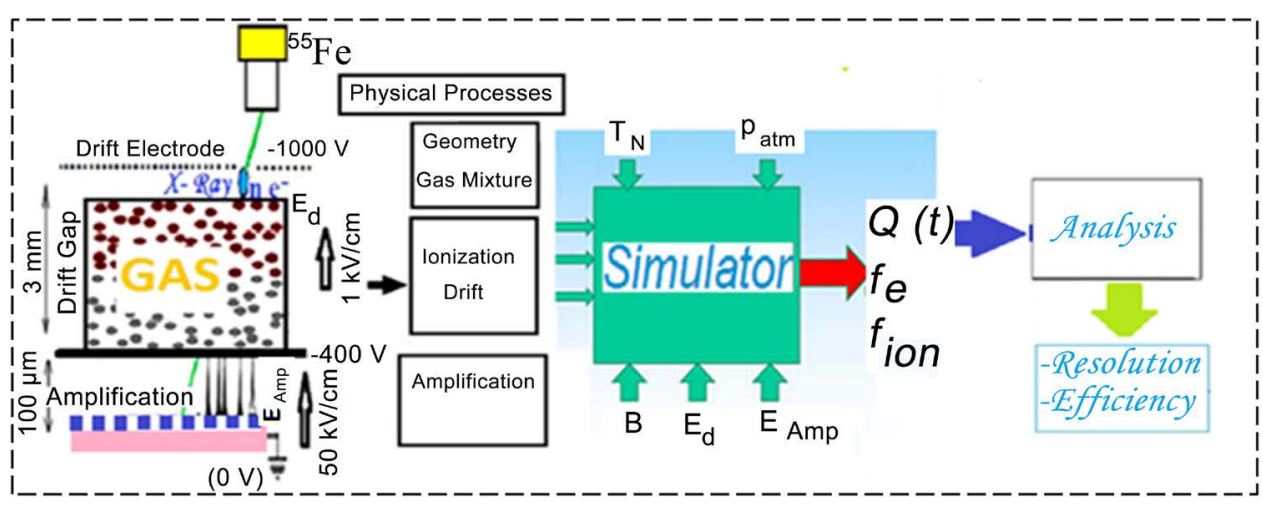

Figure 8. Principle operating and simulation tools used for MICROMEGAS detector.

Table 1. Input output parameters of micromegas detector.

\begin{tabular}{cccc}
\hline Input Parameters & Output Parameters & Gas Mixture & Radiation-Source \\
\hline Amplification Field $E_{A m p}(\mathrm{kV} / \mathrm{cm})$ & Drift Velocity $\left(v_{\mathrm{d}}\right)$ & & \\
Magnetic Field B $(\mathrm{T})$ & Longitudinal Diffusion $\left(D_{L}\right)$ & & \\
Gas Mixture & Transverse Diffusion $\left(D_{T}\right)$ & Neon-Isobutane & \\
Mixing Coefficients $\left(A_{i}, B_{i}, I=1\right.$ to 5$)$ & Charge $(Q(t))$ & $\left.\mathbf{~ N e - i C}_{\mathbf{4}} \mathbf{H}_{\mathbf{1 0}}\right)$ & ${ }^{55} \mathbf{F e} \mathbf{X}$-Rays (5.9 keV) \\
Temperature $T_{N}($ Normal Condition) & Electron Fraction Charge $\left(f_{e}\right)$ & Neon-Dimethyl-Ether & (Ne-DME) \\
Pressure $p_{a t m}$ (Atmospheric) & Ion Fraction Charge $\left(f_{\text {ion }}\right)$ & \\
Drift Field $E_{d}(\mathrm{~V} / \mathrm{cm})$ & Resolution & \\
\hline
\end{tabular}

Table 2. Mixing cefficients for different gas mixtures of an amplification gap $d=100 \mu \mathrm{m}$.

\begin{tabular}{|c|c|c|c|}
\hline Gas Mixture & Proportion (\%) & $A\left(\mathrm{~cm}^{-1}\right)$ & B $\left(\mathrm{kV} \mathrm{cm}^{-1}\right)$ \\
\hline \multirow{5}{*}{ Ne-Isobutane } & 6 & 3400 & 48.90 \\
\hline & 11 & 3700 & 56.80 \\
\hline & 20 & 4400 & 74.00 \\
\hline & 30 & 5600 & 97.80 \\
\hline & 37 & 6900 & 118.80 \\
\hline \multirow{4}{*}{ Ne-DME } & 6 & 3200 & 46.00 \\
\hline & 11 & 3300 & 50.70 \\
\hline & 30 & 3800 & 65.30 \\
\hline & 50 & 5800 & 99.80 \\
\hline
\end{tabular}

where $T_{w}$ is the first Townsend Coefficient calculated in [12], $\mathrm{n}_{0}$ is the electron number in Micro-Mesh.

The results are shown in Figure 9 and Figure 10.

First, we note that the temporal evolution of the total charge for both gas mixtures keeps the same evolution qualitatively. On the other hand, the charge decreases with the increase in the proportion of the quencher, and increases with the increase the electric field, in addition, the variation for Ne-isobutane is greater than that of Ne-DME. It is interesting to note that the total charge is simply the gain multiplied by the primary charge, and that in fact the amplified electrons do not contribute much to the final detected charge. The main contribution is clearly due to ions [15]. The proportion between the two contributions relates directly to the gain of the chamber. The fraction $f_{e}$ of the signal due to electrons is given by:

$$
f_{e}=\frac{Q_{e}}{Q_{T}}=\frac{1}{T_{w} d}\left(1-\mathrm{e}^{-T_{w} d}\right)=\frac{1}{\log (G)}
$$




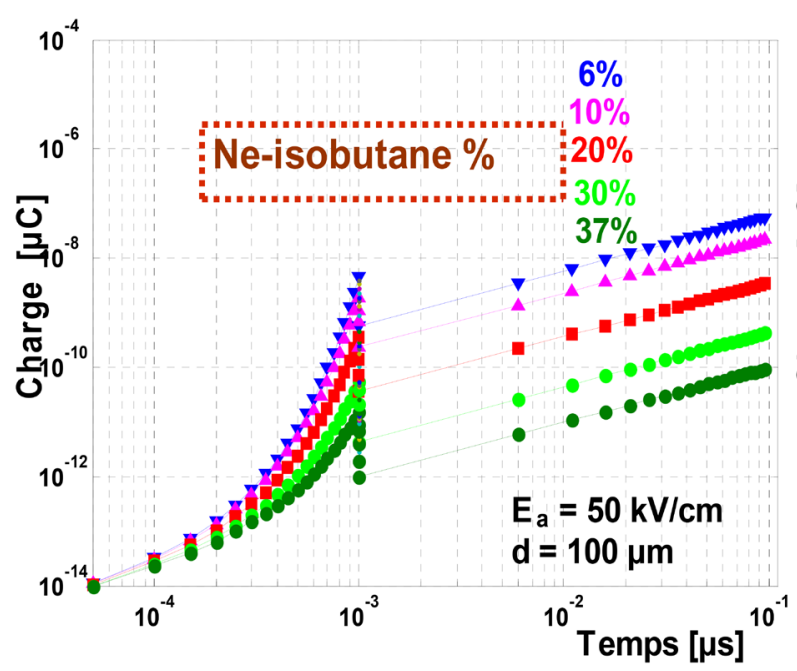

(a)

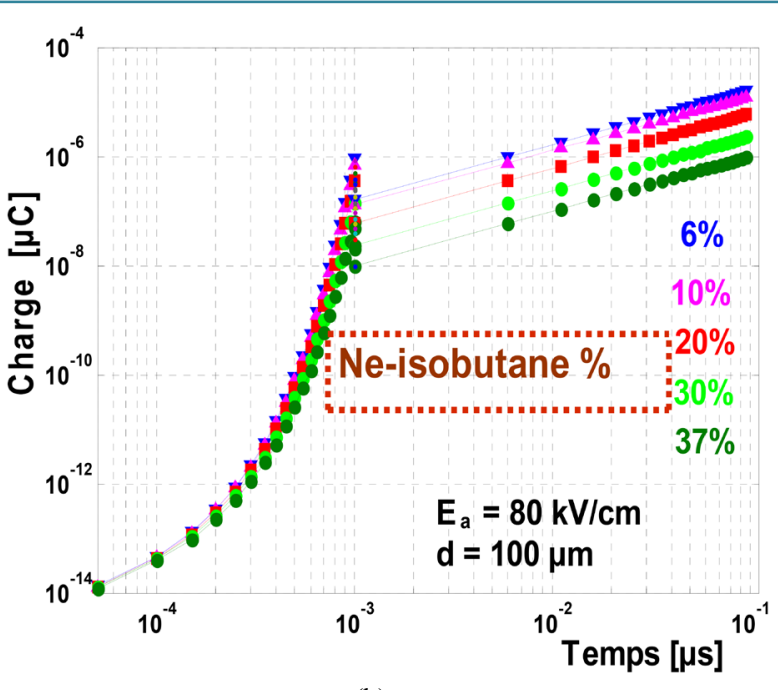

(b)

Figure 9. Charge in MICROMEGAS for different proportions of Ne-isobutane for $E_{a}=50 \mathrm{kV} / \mathrm{cm}$ (a) and $E_{a}=80 \mathrm{kV} / \mathrm{cm}$ (b).

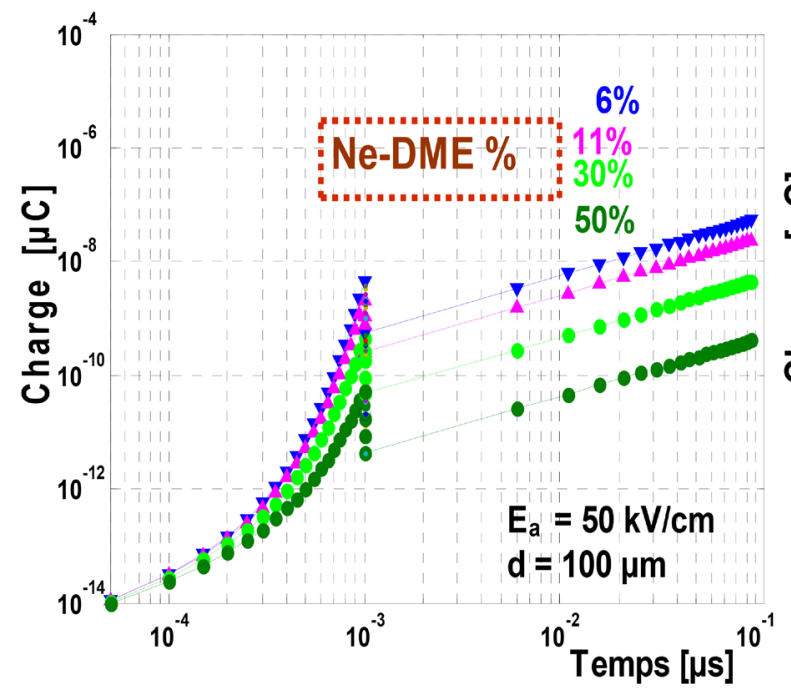

(a)

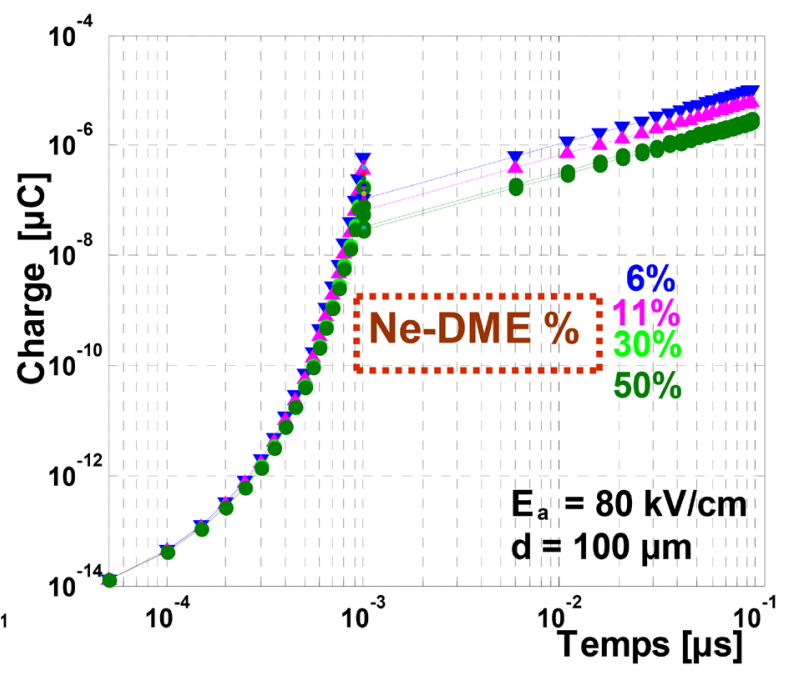

(b)

Figure 10. Charge in MICROMEGAS for different proportions of Ne-DME for $E_{a}=50 \mathrm{kV} / \mathrm{cm}$ (a) and $E_{a}=80 \mathrm{kV} / \mathrm{cm}(\mathrm{b})$.

Similarly, we can calculate the proportion of charge induced by ions:

$$
f_{\text {ion }}=1-f_{e}=1-\frac{1}{T_{w} d}\left(1-\mathrm{e}^{-T_{w} d}\right)
$$

$Q_{e}$ is the charge induced by electron.

\section{Simulation of the Electronic and Ionic Charge Fractions in MICROMEGAS}

Table 2 and the Equations (4a) and (4b) allowed us dressed a Table 3, the results of charge fractions for two electric field configurations are presented in Figure 11.

From this figure, it is found that the fraction of charge induced by the electron for each gas mixing follows an exponential law; it decreases with increasing the amplification field and the proportion of the quencher. It tends to a minimum value (saturation) until the field is very intense. However, the proportion of charge induced by ions increases with increasing the field and the quencher, it tends to a limit value in higher field. The boundary value was: 
Table 3. Induced charge fractions based in gas mixture.

\begin{tabular}{|c|c|c|c|c|c|c|c|}
\hline \multirow{2}{*}{ Mixture } & \multirow{2}{*}{ Quencher (\%) } & \multicolumn{3}{|c|}{$E_{a 1}=50 \mathrm{kV} / \mathrm{cm}$} & \multicolumn{3}{|c|}{$E_{a 2}=80 \mathrm{kV} / \mathrm{cm}$} \\
\hline & & $\boldsymbol{T}_{W i} d$ & $f_{e}(\%)$ & $f_{\text {ion }}(\%)$ & $T_{w j} d$ & $f_{e}(\%)$ & $f_{\text {ion }}(\%)$ \\
\hline \multirow{5}{*}{ Ne-isobutane } & 6 & 12.86 & 7.78 & 92.22 & 18.45 & 5.42 & 94.58 \\
\hline & 11 & 11.88 & 8.42 & 91.58 & 18.19 & 5.50 & 94.50 \\
\hline & 20 & 10.02 & 9.98 & 90.02 & 17.45 & 5.73 & 94.27 \\
\hline & 30 & 7.92 & 12.62 & 87.38 & 16.49 & 6.06 & 93.94 \\
\hline & 37 & 6.41 & 15.57 & 84.43 & 15.63 & 6.40 & 93.60 \\
\hline \multirow{4}{*}{ Ne-DME } & 6 & 12.75 & 7.84 & 92.16 & 18.01 & 5.55 & 94.45 \\
\hline & 11 & 11.97 & 8.35 & 91.65 & 17.51 & 5.71 & 94.29 \\
\hline & 30 & 10.29 & 9.71 & 90.29 & 16.80 & 5.95 & 94.05 \\
\hline & 50 & 7.88 & 12.68 & 87.32 & 16.66 & 6.00 & 94.00 \\
\hline
\end{tabular}

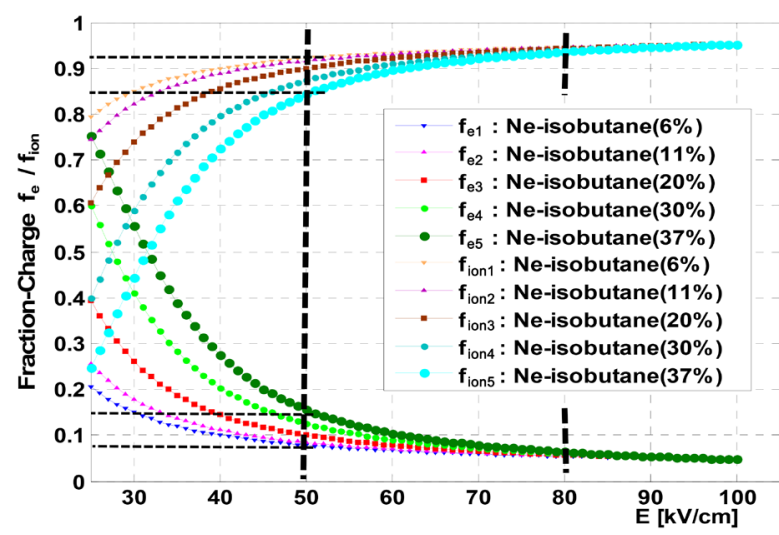

(a)

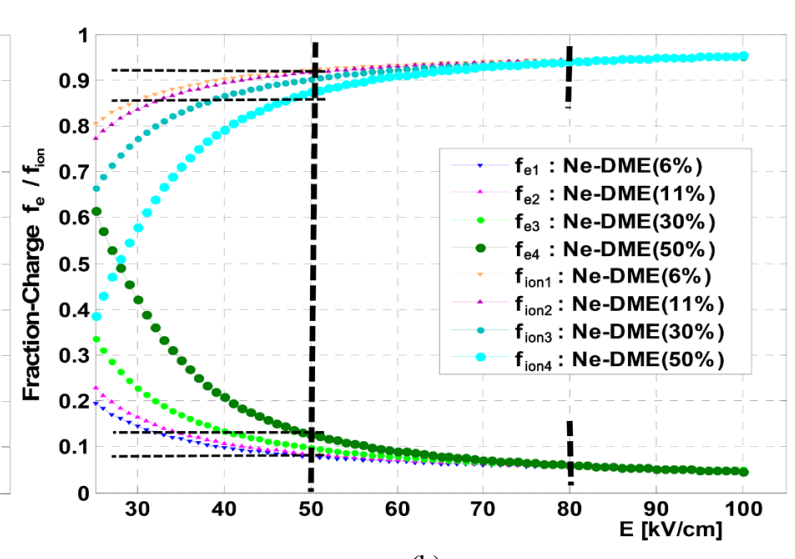

(b)

Figure 11. Induced charge fractions $\left(f_{e}\right)$ and $\left(f_{\text {ion }}\right)$ based in Ne-isobutane (a) and Ne-DME (b).

$$
\begin{aligned}
& \left\{\begin{array}{l}
\lim _{T_{w} d \rightarrow \infty} f_{e}=\frac{1}{T_{w} d} \\
\lim _{T_{w} d \rightarrow \infty} f_{\text {ion }}=1-\frac{1}{T_{w} d}
\end{array}\right. \\
& \left\{\begin{array}{l}
\lim _{T_{w} d \rightarrow 0} f_{e}=0 \\
\lim _{T_{w} d \rightarrow 0} f_{\text {ion }}=1
\end{array}, \quad T_{w} \mathrm{~d} \ll 1\right.
\end{aligned}
$$

\section{Conclusions}

The detector operating is the result of a detailed analysis of the physical phenomena. The calculus of the magnitudes characterizing this system (amplification, signal), depending on the thickness of the space, the gas mixture, geometrical configuration and the electrical distribution allowed amplification to determine the thickness of the space of the optimum gain, minimizing the effect of slight geometrical variations of the micro-grid. An amplification gap of 100 microns is optimal if it is desired to minimize the influence of the defects of planarity of the grid relative to the tracks. Simulations of $\mu$-mega focused on the study of gas mixtures. To conduct this study, we used a ${ }^{55} \mathrm{Fe}$ source emitting photons of $5.9 \mathrm{keV}$. For a gap width of 100 microns and a micro-grid prototype 500 LPI $5 \times 5 \mathrm{~cm}^{2}$, based on Ne-isobutane and Ne-DME gas mixtures. In this study, we clarified the signal (Collected charge in MICROMEGAS). In MICROMEGAS, the avalanche starts much closer to the cathode due to 
the uniform electric field in the amplification gap. This property allows MICROMEGAS to obtain faster and more intense signals improving its performance (Spatial and temporal resolutions).

In conclusion, the good agreement between experimental measurements and simulation suggests that our simulation model is all the more satisfying. However, if there are disagreements. We are tempted to interpret these observed differences, as it is in any case of systematic uncertainties in the simulation, and physical phenomena occurring within the detector were not taken into account in our simulation program: as an example, the propagation of photons in the avalanche contributing to the extension of the lateral size of the avalanche, and the Pening effect, the discharge phenomenon, ..., these phenomena are taken into account as a perspective in the future work.

In brief, through the various comparisons between real data and simulated data, we achieved a better understanding of MICROMEGAS chambers by improving its performance.

\section{References}

[1] Giomataris, Y., Rebourgeard, Ph., Robert, J.P. and Charpak, G. (1996) MICROMEGAS: A High-Granularity PositionSensitive Gaseous Detector for High Particle-Flux Environments. Nuclear Instruments and Methods, A376, 29-35.

[2] Sauli, F. (2002) Micro-Pattern Gas Detectors. Nuclear Instruments and Methods, A477, 1-7.

[3] Charpak, G., et al. (1998) First Beam Test Results with Micromegas, a High Rate, High Resolution Detector. Nuclear Instruments and Methods, A412, 47-60.

[4] Bachmann, S., Bressan, A., Ropclewski, L. and Sauli, F. (2000) High Resolution Micro-Pattern Gaseous Tracking Detectors. CERN, Nuclear Physics A663\&664, 1069c-1072c.

[5] Giomataris, Y. (1998) Development and Prospects of the New Gaseous Detector MICROMEGAS. Nuclear Instruments and Methods, A419, 239. http://dx.doi.org/10.1016/S0168-9002(98)00865-1

[6] Sarvestani, A., et al. (1997) Thin-Gap Parallel Plate Chamber Variation. Nuclear Instruments and Methods, A410, 238.

[7] Barrouch, G., et al. (1999) Development of a Fast Gaseous Detector: “Micromegas”. Nuclear Instruments and Methods, A423, 32-48.

[8] Korff, S.A. (1955) Electrons and Nuclear Counters. Van Nostrand, Princeton.

[9] Hamid, M., Seddik, B. and Abdelrhani, N. (2013) Micromegas Detector: Modeling Large Families Based on Gaseous Mixtures. LAP LAMBERT, Academic Publishing.

[10] Mounir, H., Bri, S. and Haddad, M. (2012) Micromegas Signals Produced in Micromesh Based in Argon-Isobutane (Ar-Isobutane) and Argon-Dimethyl-Ether (Ar-DME) Gas Mixtures. European Journal of Scientific Research, 81, 465475.

[11] Puil Geal, M. (2000) The Development of MICROMEGAS, a New Detector Gaseous Positron Micro-Grid. Ph.D. Thesis, University of Caen, Caen.

[12] Mounir, H. and Bri, S. (2013) Micromegas Detector Using 55Fe X-ray Source. International Journal of Advanced Scientific and Technical Research, 1, 671-684.

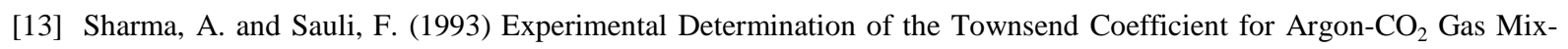
tures at High Fields. Nuclear Instruments and Methods, A334, 420-424.

[14] Nakhostin, M., Baba, M., Ohtsuki, T., Oishi, T. and Itoga, T. (2007) Precise Measurement of First Townsend Coefficient, Using Parallel Plate Avalanche Chamber. Nuclear Instruments and Methods in Physics Research, A572, 9991003.

[15] Chefdeville, M. (2009) Development of Micromegas-Like Gaseous Detectors Using a Pixel Readout Chip as Collecting Anode. Thesis, University of Amsterdam, Amsterdam. 
Scientific Research Publishing (SCIRP) is one of the largest Open Access journal publishers. It is currently publishing more than 200 open access, online, peer-reviewed journals covering a wide range of academic disciplines. SCIRP serves the worldwide academic communities and contributes to the progress and application of science with its publication.

Other selected journals from SCIRP are listed as below. Submit your manuscript to us via either submit@scirp.org or Online Submission Portal.
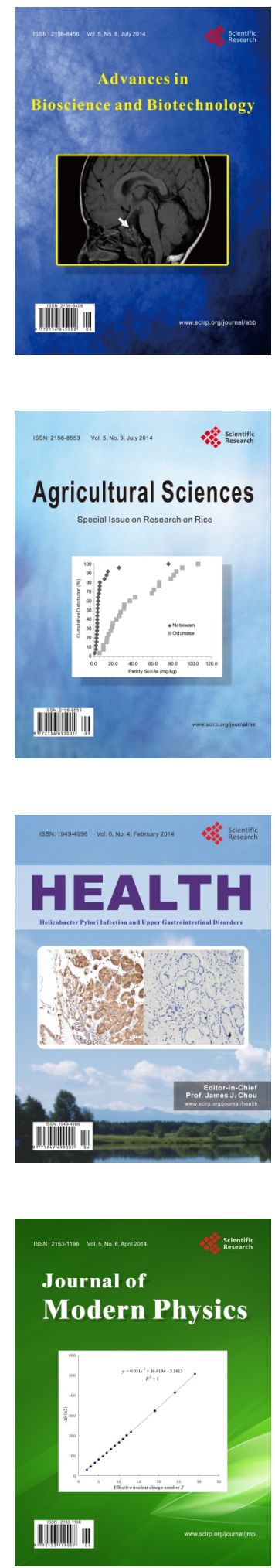
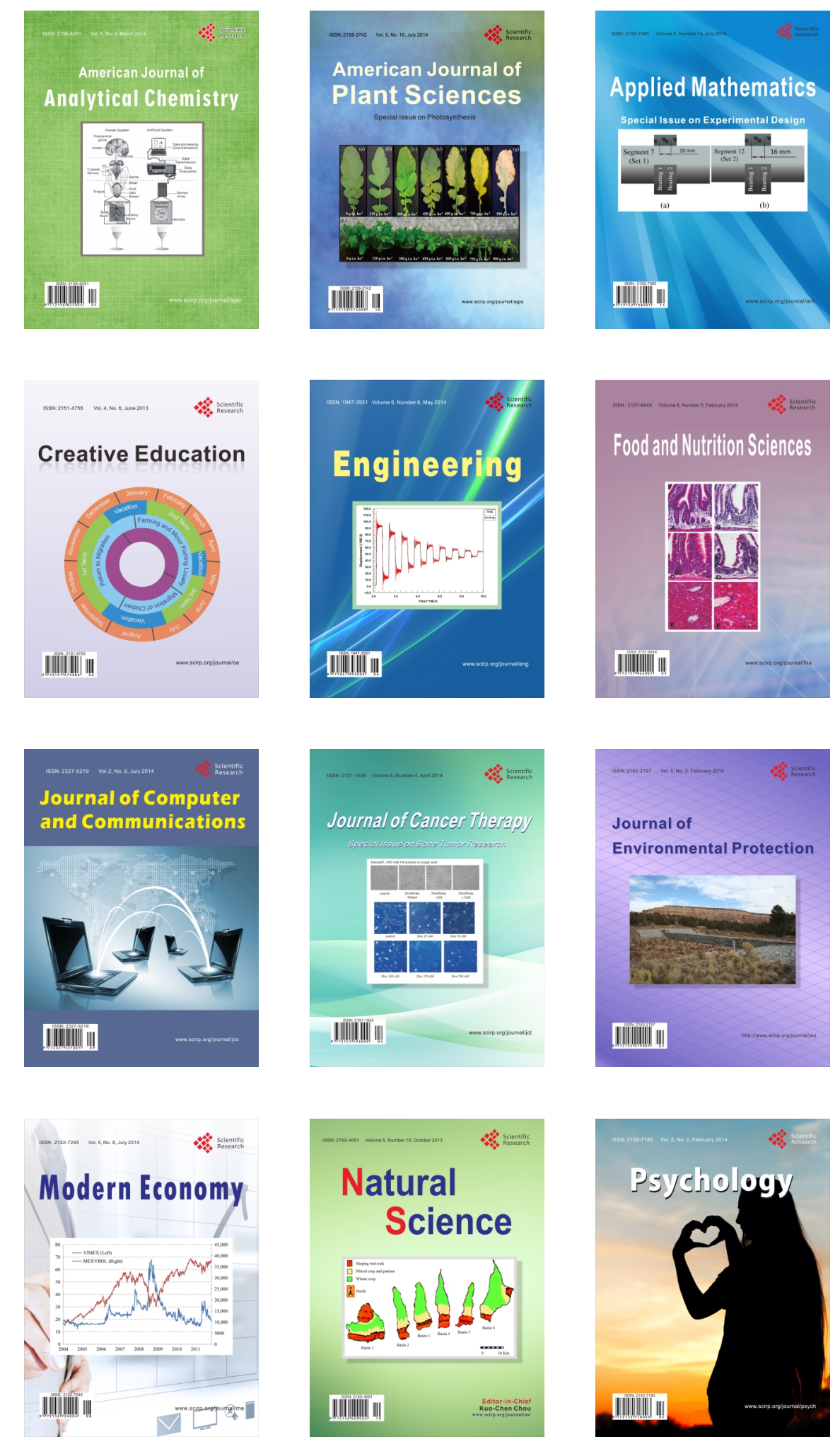